\title{
VARIATIONAL-LAGRANGIAN IRREVERSIBLE THERMODYNAMICS OF INITIALLY-STRESSED SOLIDS WITH THERMOMOLECULAR DIFFUSION AND CHEMICAL REACTIONS
}

\author{
By M. A. Biot \\ Royal Academy of Belgium, Brussels, Belgium
}

(Received 7th February 1977)

\begin{abstract}
SUMMARY
A GENERAL principle of virtual dissipation in irreversible thermodynamics is applied to a solid under initial stress with small non-isothermal incremental deformations and coupled thermomolecular diffusion and chemical reactions. Dynamical field and Lagrangian equations are obtained directly by variational procedures. In addition, the treatment embodies new fundamental concepts and methods in the thermodynamics of open systems and thermochemistry. The new concept of 'thermobaric potential' is briefly outlined. The theory is also applicable to porous solids with 'diffusionlike' behaviour of pore-fluid mixtures. General validity of viscoelastic correspondence for chemical or other relaxation processes with internal coordinates is indicated in acoustic propagation and seismic problems.
\end{abstract}

\section{INTRODUCTION}

VARIATIONAL principles and a corresponding Lagrangian approach to irreversible thermodynamics (BIOT, 1954, 1955) were originally developed in the linear context. This includes the particular case of a continuum under initial stress as treated extensively by the writer in a monograph (BIOT, 1965). Our purpose here is to extend the theory to an initially-stressed continuum including heat conduction, molecular diffusion of various substances in solution in the solid, and multiple chemical reactions.

The variational principle which actually constitutes a generalization of d'Alembert's principle to irreversible thermodynamics is also applicable to non-linear systems as shown more recently (Brot, 1975, 1976b). It achieves a synthesis between thermodynamics and classical mechanics. However, in the present theory it will be sufficient to use the principle in its earlier form in the linear context.

The formulation of the variational principle introduced a non-classical 'collective potential' which has been used repeatedly in many applications, including the treatment of piezoelectric crystals (MINDLIN, 1961, 1974).

The problems cited above did not involve molecular diffusion or chemical reactions. In order to deal with these problems, a new concept was developed, referred to as the 'Thermobaric potential' (BIOT, 1976a). Its derivation is briefly outlined in Section 3. 
The concepts of incremental strain with local rotation, incremental stress and collective potential for a continuum initially in thermodynamic equilibrium under initial stress are developed in Sections 4, 5 and 6 . In the definition of strain, attention is called to the importance of separating the deformation from the rotation and to a non-tensorial definition. The entropy production based on ONSAGER's (1931) principle is evaluated in Section 7 for coupled thermomolecular diffusion.

The field equations are derived directly from the variational principle in Section 8 . The differential equations obtained are completely general and govern the dynamics of the small perturbations of a continuum under initial stress with thermomolecular diffusion and in the presence of a potential body force field. They constitute a new result. Derivation of such equations directly from a single physical and general variational principle is in contrast with current procedures which generally require a knowledge of the differential field equations in order to establish the variational properties in each case.

Lagrangian equations with generalized coordinates are obtained also directly from the variational principle in Section 9. The generalized boundary driving forces are of mixed mechanical and thermodynamic nature. Basic reciprocity relations are implicit in these equations valid for all kinds of systems and boundary conditions. They constitute a general law for all linear thermodynamic systems and do not require a special derivation for each particular problem, as wrongly assumed by many workers.

The results are generalized to include chemical reactions in Section 10 which embodies a new treatment of physical chemistry and of the chemical affinity (BIOT, 1976a,1977). The acoustic propagation is discussed in Section 11 from the viewpoint of internal coordinates with chemical or other relaxation effects such as microphase changes. A generalized form of the principle of viscoelastic correspondence is formulated.

Wave propagation in solids with chemical reactions has been treated by Nunziato (1973) without initial stress and diffusion and by a more traditional type of chemical thermodynamics.

Attention is called to the applicability of the present theory to porous solids in those cases where the diffusion of a fluid mixture through the pores behaves as a molecular diffusion.

\section{Collective Potential of a Cell}

Consider a homogeneous element which is initially a unit cube under the initial stresses $S_{i j}$. With cube edges along Cartesian axes, $S_{i j}$ are the force components acting on the faces. The cube is at uniform temperature. This solid element will be called a primary cell $C_{\mathrm{p}}$. We shall assume that it may lose or acquire matter in the form of pure substances in solution. Hence it is an open thermodynamic system. A new approach to such systems has recently been developed which we shall briefly outline here.

We adjoin to the primary cell a set of large rigid supply cells $C_{\mathrm{sk}}$ each containing a pure substance $k$. The supply cells are all at the same pressure $p_{0}$ and temperature $T_{0}$. As shown earlier (BIOT, 1976a,1977), the choice of the same pressure and temperature 
for all supply cells is required to avoid the Gibbs paradox. We also adjoin a large isothermal reservoir at the temperature $T_{0}$ which we have called a thermal well. The system constituted by the primary cell $C_{\mathrm{p}}$, the supply cells $\sum^{k} C_{\mathrm{s} k}$, and the thermal well has been called a hypersystem.

This hypersystem is now assumed to undergo a reversible transformation produced by external forces. In this transformation, heat and matter are transported reversibly within the hypersystem. No heat or matter are exchanged with the environment. The work performed on the system includes the work of reversible heat pumps to transfer heat between cells at different temperatures. We denote by $\mathscr{V}$ the total reversible work on the hypersystem. The first principle of thermodynamics yields the relation

$$
\mathscr{V}=\mathscr{U}+H_{0},
$$

where $\mathscr{U}$ is the internal energy of the system of cells $C_{\mathrm{p}}+\sum^{k} C_{\mathrm{s} k}$ and $H_{0}$ is the heat energy acquired by the thermal well.

An important property of $\mathscr{V}$ is obtained by considering that the transformation is reversible; and hence the total entropy of the hypersystem remains unchanged and may be put equal to zero. We write

$$
\mathscr{S}+H_{0} / T_{0}=0,
$$

where $\mathscr{S}$ is the collective entropy of the system $C_{\mathrm{p}}+\sum^{k} C_{\mathrm{s} k}$. Elimination of $H_{0}$ between (2.1) and (2.2) yields

$$
\mathscr{V}=\mathscr{U}-T_{0} \mathscr{S} .
$$

The state variables of the supply cells are the masses $m_{k}$ of the various substances extracted from these cells and transferred to the primary cell. Since the $m_{\mathrm{k}}$ are also part of the state variables of the primary cells, the latter completely determine the values of $\mathscr{U}$ and $\mathscr{S}$. Hence, $\mathscr{V}$ is a function only of the state variables of the primary cell. It is therefore possible to refer to $\mathscr{V}, \mathscr{U}$ and $\mathscr{S}$ as the collective potential, collective energy and collective entropy of the primary cell $C_{\mathrm{p}}$.

\section{Thermobaric Potential: a New Concept}

In its initial state, the cell $C_{\mathrm{p}}$ is assumed to be a cube of unit size. The deformation is homogeneous and defined by six independent variables $\varepsilon_{i j}=\varepsilon_{j i}$ which are not necessarily tensor components. Their definition will be discussed in Section 4 . The corresponding stress components $\tau_{i j}=\tau_{i j}$ are defined by the work $\tau_{i j} d \varepsilon_{i j}$ accomplished in obtaining any arbitrary strain differential. Assume that the cell undergoes such a differential deformation, while masses $d m_{k}$ are injected reversibly into it and a differential amount of heat is absorbed. This change being accomplished reversibly, the concentrations of the pure substances and the temperature $T$ of the cell are assumed to remain uniform. By definition, the increase in the collective cell potential is

$$
d \mathscr{V}=\tau_{i j} d \varepsilon_{i j}+\sum^{k} \psi_{k} d m_{k}+\theta d s_{T}
$$


We put

$$
\theta=T-T_{0}, \quad d s_{T}=d h / T_{0} .
$$

In equation (3.1) and all subsequent ones we drop the summation sign for indices except those of $m_{k}$.

The term $\theta d s_{T}$ is the work required by a heat pump extracting heat at the temperature $T_{0}$ from the thermal well and injecting an amount $d h$ in the primary cell at the temperature $T$.

The quantity $\psi_{k}$ represents the reversible work required to extract a unit mass of substance $k$ from the supply cell $C_{\text {sk }}$ to bring it into thermodynamic equilibrium at pressure $p_{k}$ and temperature $T$ with the primary cell $C_{\mathrm{p}}$ and to inject this mass into the primary cell. We have called $\psi_{k}$ the thermobaric potential, and referred to the mass transport as a thermobaric transfer. Although this definition is quite general, we shall assume that the pure substances are fluids. In this case, $p_{k}$ is the pressure of the substance in equilibrium with the primary cell through a semi-permeable membrane. By definition, we have called $p_{k}$ the partial pressure of the pure substance in the primary cell. The value of $\psi_{k}$ obtained earlier (Brot, 1976a,1977) is

$$
\psi_{k}\left(p_{k}, T\right)=\int_{p_{0} T_{0}}^{p_{k} T}\left(\frac{d p_{k}}{\rho_{k}}+\theta d \bar{s}_{k}\right),
$$

where $d \bar{s}_{k}$ is the entropy differential of a unit mass of pure substance. The first term represents the work accomplished during thermobaric transfer by the varying pressure $p_{k}$ acting on the fluid at various corresponding densities $\rho_{k}$. This is verified by integration by parts of this term which yields three terms and bring out the work of extraction from $C_{\mathrm{s} k}$ and injection into $C_{\mathrm{p}}$. The term $\theta d \bar{s}_{k}$ is the work accomplished by the heat pumps at every step for each intermediate temperature $\theta$. The integral (3.3) is independent of the path of integration. The entropy of a unit mass of pure substance $k$ in equilibrium with the primary cell is

$$
\bar{s}_{k}=\int_{p_{0} T_{0}}^{p_{k} T} d \bar{s}_{k}
$$

We shall call $\bar{s}_{k}$ the specific relative entropy of the pure substance in the primary cell.

Injecting into the primary cell, the mass $d m_{k}$ in equilibrium with it does not produce any entropy. Hence, the increase of collective entropy of the primary cell is

$$
d \mathscr{P}=\sum^{k} \vec{s}_{k} d m_{k}+d s_{T}
$$

where the first term is due to convection and $d s_{T}$ is due to heat conduction.

\section{InCREMENTAL STRAIN}

The definition of stress by means of the virtual work $\tau_{i j} \delta \varepsilon_{i j}$ is quite general and $\varepsilon_{i j}$ may be Green's strain tensor. However, in problems of incremental stresses, it is essential to avoid spurious complications which arise from the use of Green's tensor. This is accomplished by using a definition of strain introduced by the writer in 1939 


\section{ERRATA}

\section{VARIATIONAL-LAGRANGIAN IRREVERSIBLE THERMODYNAMICS OF INITIALLY-STRESSED SOLIDS WITH THERMOMOLECULAR DIFFUSION AND CHEMICAL REACTIONS}

By M. A. BioT

J. Mech. Phys. Solids 25, 289 (1977)

p. 293. In equation $\left(4.7_{1}\right)$, read

$$
\eta_{11}=\frac{1}{2} a_{21}^{2} .
$$

p. 294. On the second line after equation (5.4), read

Since $\varepsilon_{i j}, m_{k}$ and $\mathscr{P}$ are state variables, ...

p. 297. In equation (6.15), replace

$$
\sum^{k} \mathscr{G}\left(\xi_{i}\right) \delta m_{k} \quad \text { by } \quad \sum^{k} \delta\left[\mathscr{G}\left(\xi_{i}\right) m_{k}\right]
$$

Equations (6.16) should read

$$
\frac{\partial \mathscr{G}\left(\xi_{i}\right)}{\partial \xi_{i}}-\frac{\partial \mathscr{G}\left(x_{i}\right)}{\partial x_{i}}=\mathscr{G}_{i j} u_{j}, \quad \mathscr{G}\left(\xi_{i}\right)=\mathscr{G}_{i} u_{i}+\mathscr{G}\left(x_{i}\right) .
$$

In equation (6.18), replace

$$
\sum^{k} \mathscr{G}_{i} m_{k} u_{i} \quad \text { by } \quad \sum^{k} \mathscr{G}_{i} \delta\left(m_{k} u_{i}\right) .
$$

p. 299. In equation (7.9), replace

$$
\sum^{k} \mathscr{C}_{i j}^{k} M_{i}^{k} S_{j}^{T} \quad \text { by } \quad \sum^{k} \mathscr{C}_{i j}^{k} \dot{M}_{i}^{k} \dot{S}_{j}^{T} .
$$

p. 300 . In equation $(8.6)$, replace

$$
\rho \ddot{u}_{i} \delta u_{i} \quad \text { by } \quad\left(\rho \ddot{u}_{i}+\sum^{k} m_{0 k} a_{i}^{k}\right) \delta u_{i} .
$$

In equation (8.9), replace

$$
\delta \dot{M}_{i}^{k} \quad \text { by } \quad \delta M_{i}^{k} .
$$

p. 306. In equation $\left(11.6_{1}\right)$, read

$$
e_{i j}=\frac{1}{2}\left(\frac{\partial u_{i}}{\partial x_{j}}+\frac{\partial u_{j}}{\partial x_{i}}\right)
$$


and developed extensively in a book (BIOT, 1965). In this definition, we start with a linear transformation of coordinates

$$
\xi_{i}=\left(\delta_{i j}+\varepsilon_{i j}\right) x_{j}
$$

with six independent coefficients $\varepsilon_{i j}=\varepsilon_{j i}$ which are chosen to represent the finite strain. The linear transformation (4.1) is then followed by a solid rotation and the resultant total transformation becomes

$$
\xi_{i}=\left(\delta_{i j}+a_{i j}\right) x_{j} .
$$

The $\varepsilon_{i j}$ 's are functions of the nine coefficients $a_{i j}$. To the second order, we have shown

where

$$
\varepsilon_{i j}=e_{i j}+\eta_{i j}
$$

$$
\eta_{i j}=\frac{1}{2}\left(e_{k j} \omega_{k i}+e_{k i} \omega_{k j}+\omega_{k i} \omega_{k j}\right)
$$

and

$$
e_{i j}=\frac{1}{2}\left(a_{i j}+a_{j i}\right), \quad \omega_{i j}=\frac{1}{2}\left(a_{i j}-a_{j i}\right) .
$$

Actually, the procedure is general and yields $\varepsilon_{i j}$ to any order.

As pointed out (BIOT, 1965, p. VII and 1973, p. 483) this definition is quite flexible and not unique. For example, in two dimensions we may start from the linear transformation

$$
\left.\begin{array}{l}
\xi_{1}=\left(1+\varepsilon_{11}\right) x_{1}+2 \varepsilon_{12} x_{2}, \\
\xi_{2}=\left(1+\varepsilon_{22}\right) x_{2}
\end{array}\right\}
$$

and choose the three coefficients $\varepsilon_{11}, \varepsilon_{22}, \varepsilon_{12}=\varepsilon_{21}$ as non-tensorial components of strain. To the second order, it has been shown (BIOT, 1974) that $\varepsilon_{i j}$ is given by (4.3) with

$$
\left.\begin{array}{c}
\eta_{11}=\frac{1}{2} a_{22}^{2}, \quad \eta_{22}=-\frac{1}{2} a_{21}\left(2 a_{12}+a_{21}\right), \\
\eta_{12}=\eta_{21}=\frac{1}{2} a_{21}\left(a_{22}-a_{11}\right) .
\end{array}\right\}
$$

Similar non-tensorial definitions may be obtained in three dimensions (BIor, 1973).

This leads also to the non-tensorial concept of slide modulus which is cssential in the theory of initially-stressed media (BIOT, 1965) - a point which seems to have been overlooked in the current literature.

When dealing with a non-homogeneous deformation the transformation (4.2) is replaced by a linear transformation between differentials $d \xi_{i}$ and $d x_{i}$, and the coefficients $a_{i j}$ become

$$
a_{i j}=\partial u_{i} / \partial x_{j},
$$

where $u_{i}=\xi_{i}-x_{i}$ is the displacement field, and $x_{i}$ are the initial coordinates.

\section{InCRemental Cell Potential}

A unit cube element of the solid under initial stress $S_{i j}$ at the temperature $T_{0}$ is subjected to small perturbations, represented by small strains $\varepsilon_{i j}$, small increases 
$m_{k}$ of masses in solution, and a small increase in temperature $\theta$. We put

$$
\tau_{i j}=S_{i j}+t_{i j}, \quad \psi_{k}=\psi_{0 k}+\Delta \psi_{k},
$$

where $t_{i j}$ is the incremental stress per unit initial area and referred to locally rotated axes. Also,

$$
\psi_{0 k}=\int_{p_{0} T_{0}}^{p_{0 k} T_{0}} \frac{d p_{k}}{\rho_{k}}
$$

is the value of $\psi_{k}$ in the initial state, where $p_{0 k}$ is the partial pressure of substance $k$ in this state. To the first order, we also write

$$
\Delta \psi_{k}=\int_{p_{0 k} T_{0}}^{p_{k} T} \frac{d p_{k}}{\rho_{k}}
$$

Again, if we neglect second-order quantities, then (3.5) becomes

$$
\mathscr{S}=\sum^{k} \bar{s}_{0 k} m_{k}+s_{T}
$$

where $\bar{s}_{0 k}$ is the initial relative specific entropy and $s_{T}$ is the increase of entropy due to the heat supplied by conduction. Since $\varepsilon_{i j}, m_{k}$ and $f($ are state variables, (5.4) leads to the important conclusion that $\varepsilon_{i j}, m_{k}$ and $s_{T}$ may be chosen as state variables. The fact that $s_{T}$ is now a state variable leads to considerable simplification. This is a consequence of the linearization and is not true in the non-linear theory.

For convenience, we shall drop the word 'collective' to designate the cell potential $\mathscr{V}$. With the values (5.1) for $\tau_{i j}$ and $\psi_{k}$, its differential (3.1) becomes

$$
d \mathscr{V}=d v+S_{i j} d \varepsilon_{i j}+\sum^{k} \psi_{0 k} d m_{k}
$$

where

$$
d v=t_{i j} d e_{i j}+\sum^{k} \Delta \psi_{k} d m_{k}+\theta d s_{T} .
$$

In the linearized theory, we need evaluate $\mathscr{V}$ only to the second order. Hence, in the value of $v$ we have replaced $t_{i j} d \varepsilon_{i j}$ by $t_{i j} d e_{i j}$. Although $\mathscr{V}$ appears to represent an incremental potential this is not a true local concept. Actually, it is preferable to refer to $v$ as the local incremental potential. This will be justified in Section 6 by showing that $v$ embodies true local properties. If the material is locally stable, physically this leads to the important conclusion that $v$ is positive definite. Equations (5.5) and (5.6) generalize the expression derived earlier in the purely thermoelastic context (BIOT, 1973). The incremental cell potential $v$ is a quadratic function of the state variables $e_{i j}, m_{k}$ and $s_{T}$ with the properties

$$
\frac{\partial v}{\partial e_{i j}}=t_{i j}, \quad \frac{\partial v}{\partial m_{k}}=\Delta \psi_{k}, \quad \frac{\partial v}{\partial s_{T}}=\theta .
$$

Since the value of $v$ is independent of the path of integration, it is convenient to integrate (5.6) first at constant temperature $(\theta=0)$. This yields a value of $v$ at $\theta=0$, viz.

$$
\bar{v}=\frac{1}{2} C_{i j}^{\mu v} e_{i j} e_{\mu v}+\sum^{k} C_{i j}^{k} e_{i j} m_{k}+\frac{1}{2} \sum^{k l} C^{k l} m_{k} m_{l}
$$


with the properties

$$
\left.\begin{array}{c}
C_{i j}^{\mu \mathrm{v}}=C_{j i}^{\mu \nu}=C_{i j}^{p \mu}=C_{\mu 0}^{i j}, \\
C_{i j}^{k}=C_{j i}^{k}, \quad C^{k l}=C^{l k} .
\end{array}\right\}
$$

We then integrate (5.6) by varying the temperature with $d e_{i j}=d m_{k}=0$. Since $d s_{T}=\left(c / T_{0}\right) d \theta$, we derive

$$
v=\bar{v}+\frac{1}{2} \frac{c \theta^{2}}{T_{0}}
$$

where $c$ is the heat capacity of the cell per unit volume for $d e_{i j}=d m_{k}=0$. Hence, by neglecting higher order quantities, $c$ is the heat capacity in the initial state $\left(e_{i j}=m_{k}=\theta=0\right)$.

We may also express $s_{T}$ to the first order as

$$
s_{T} T_{0}=\gamma_{i j} e_{i j}+\sum^{k} h^{k} m_{k}+c \theta .
$$

The coefficient $\gamma_{i j}=\gamma_{j i}$ is the heat absorbed at constant composition and temperature $\left(m_{k}=\theta=0\right)$ per unit strain $e_{i j}$. The coefficient $h^{k}$ is the heat absorbed when a unit mass of $m_{k}$ is injected reversibly at $e_{i j}=\theta=0$. Solving (5.11) for $\theta$ and substituting into (5.10) yields $v$ as a quadratic form in $e_{i j}, m_{k}$ and $s_{T}$. We then obtain $t_{i j}, \Delta \psi_{k}$ and $\theta$ as linear functions of $e_{i j}, m_{k}$ and $s_{T}$ by applying (5.7).

A physical interpretation of the coefficients in expression (5.8) is easily obtained. For $m_{k}=0$, we have

$$
t_{i j}=C_{i j}^{\mu v} e_{\mu v}
$$

Hence, $C_{i j}^{\mu \nu}$ are the incremental elastic coefficients at constant composition and temperature $\left(m_{k}=\theta=0\right)$. Also, at constant temperature $(\theta=0)$ we derive

$$
\frac{\partial \bar{v}}{\partial m_{k}}=\int_{p_{0 k} T_{0}}^{p_{k} T_{0}} \frac{d p_{k}}{\rho_{k}}=\frac{p_{k}-p_{0 k}}{\rho_{0 k}}=C_{i j}^{k} e_{i j}+\sum C^{k l} m_{l},
$$

where $\rho_{0 k}$ is the density of the pure substance at the initial temperature $T_{0}$ and partial pressure $p_{0 k}$. Hence, the coefficients on the right-hand side may be interpreted in terms of increments of partial pressure $p_{k}-p_{0 k}$.

\section{Collective Potential of an Initially Stressed Continuum. EQUILIBRIUM CONDITION}

An important property of the collective potential is additivity. Hence, for a finite continuum occupying an initial domain $\Omega$, its collective potential is

$$
V=\int_{\Omega} \mathscr{r} d \Omega
$$

where $d \Omega=d x_{1} d x_{2} d x_{3}$ is the volume element in the initial coordinates $x_{i}$. The displacement field of the solid is $u_{i}=\xi_{i}-x_{i}$ and $\mathscr{V}$ is the local cell potential per unit volume. The collective energy and entropy of the system are

$$
U=\int_{\Omega} \mathscr{U} d \Omega, \quad S=\int_{\Omega} \mathscr{S} d \Omega,
$$

where $\mathscr{U}$ and $\mathscr{S}$ are the collective energy and entropy per unit initial volume as 
defined in Section 2 to include the contribution of the supply cells. From (2.3), (6.1) and (6.2), we derive

$$
V=U-T_{0} S .
$$

If heat and matter are exchanged only within the continuum, then the supply cells remain unchanged. Conservation of energy requires

$$
U=W,
$$

where $W$ is the work performed by external forces on the continuum. With this value for $U$, equation (6.3) becomes

$$
V-W=-T_{0} S^{\prime}
$$

We have written $S^{\prime}$ instead of $S$ because it now represents the total entropy produced in the continuum. This relation is the same as derived earlier (Biот, 1955). In differential or variational form, equation (6.5) is written

$$
\delta V-\delta W=-T_{0} \delta S^{\prime}
$$

If the continuum lies in a potential body force field, such as gravity, then we may write the variational equation (6.6) as

$$
\delta(V+G)-\delta W^{\prime}=-T_{0} \delta S^{\prime},
$$

where $G$ is the potential energy in the body force field and $W^{\prime}$ is the work performed by other forces. The potential energy $G$ is

$$
G=\int_{\Omega}\left(\rho+\sum^{k} m_{k}\right) \mathscr{G}\left(\xi_{i}\right) d \Omega .
$$

In this expression, $\rho$ is the density at the initial point $x_{i}$ in the initial state, $m_{k}$ are the masses added per unit initial volume at the displaced point $\xi_{i}$, and $\mathscr{G}\left(\xi_{i}\right)$ is the body force potential per unit mass at the displaced point $\xi_{i}$.

We shall now transform the variational equation (6.7) taking into account the initial equilibrium of the continuum. This is expressed by stating that there is no virtual production of entropy $\left(\delta S^{\prime}=0\right)$ for variations with suitable conservation constraints on the variables $u_{i}, m_{k}$ and $s_{T}$ in the initial state.

Since $\delta v=0$ in the initial state, the variation $\delta V$ derived from (5.5) and (6.1) becomes

$$
\delta V_{\mathrm{eq}}=\int_{\mathbf{\Omega}} \delta \mathscr{V}=\int_{\mathbf{\Omega}}\left(S_{i j} \delta e_{i j}+\sum^{k} \psi_{0 k} \delta m_{k}\right) d \Omega
$$

Also, around the equilibrium state, the variation $\delta G$ is

$$
\delta G_{\mathrm{eq}}=\int_{\Omega}\left[\rho \frac{\partial \mathscr{G}\left(x_{i}\right)}{\partial x_{i}} \delta u_{i}+\sum^{k} \mathscr{G}\left(x_{i}\right) \delta m_{k}\right] d \Omega .
$$

The virtual work of the tractions $f_{i}$ applied at the boundary $A$ in the initial state is

$$
\delta W_{\mathrm{eq}}^{\prime}=\int_{A} f_{i} \delta u_{i} d A .
$$

The equilibrium condition in the initial state is obtained by putting $\delta S^{\prime}=0$ in (6.7). 
With the values (6.9), (6.10) and (6.11) for $\delta V_{\text {eq }}, \delta G_{\text {eq }}$ and $\delta W_{\text {eq }}^{\prime}$ this condition is

$$
\delta V_{\mathrm{eq}}+\delta G_{\mathrm{eq}}-\delta W_{e q}^{\prime}=0 .
$$

By subtracting (6.12) from (6.7) we obtain

$$
\delta \int_{\Omega}\left(v+S_{i j} \eta_{i j}\right) d \Omega+\delta G-\delta G_{\mathrm{eq}}-\delta W_{\mathrm{in}}^{\prime}=-T_{0} \delta S^{\prime}
$$

where

$$
\delta W_{\mathrm{ln}}=\delta W^{\prime}-\int_{A} f_{l} \delta u_{l} d A
$$

is the work of incremental forces. The variational equation (6.13) now applies to non-equilibrium conditions. We may write the value of $\delta G$ more explicitly as

$$
\delta G=\int_{\mathbf{\Omega}}\left[\rho \frac{\partial \mathscr{G}\left(\xi_{i}\right)}{\partial \xi_{i}} \delta u_{i}+\sum_{\Lambda}^{k} \xi_{\mathcal{G}}\left(\xi_{i}\right) \not m_{k}\right] d \Omega
$$

To the first order, we write

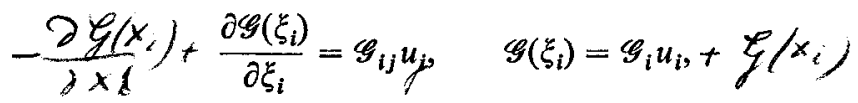

with

Hence,

$$
\mathscr{G}_{i j}=\frac{\partial^{2} \mathscr{G}\left(x_{i}\right)}{\partial x_{i} \partial x_{j}}, \quad \mathscr{G}_{i}=\frac{\partial \mathscr{G}\left(x_{i}\right)}{\partial x_{i}}
$$

$$
\delta G-\delta G_{\mathrm{eq}}=\int_{\Omega}\left(\rho \mathscr{G}_{i j} u_{j} \delta u_{i}+\sum^{k} \mathscr{G}_{i} m_{k} u_{i}\right) d \Omega
$$

By introducing this value into (6.13) we obtain the variational relation

where

$$
\delta \mathscr{P}-\delta W_{\text {in }}=-T_{0} \delta S^{\prime},
$$

$$
\mathscr{P}=\int_{\mathbf{\Omega}}\left(v+S_{i j} \eta_{i j}+\frac{1}{2} \rho \mathscr{G}_{i j} u_{i} u_{j}+\sum^{k} \mathscr{G}_{i} m_{k} u_{i}\right) d \Omega .
$$

As mentioned previously, the incremental potential $v$ represents a local thermodynamic property since the other terms are either due to the body force field or can be made to vanish locally $\left(\eta_{i j}=0\right)$ by a suitable rotation of the coordinate axes.

Expression (6.20) for the collective potential of an initially-stressed system is a generalization of expressions derived earlier for an isothermal or a thermoelastic medium (BIOT, 1965, 1973). Note that for a uniform gravity field, $\mathscr{G}_{i j}=0$. If incremental boundary tractions $\Delta f_{i}$ are applied per unit initial area, then the incremental work (6.14) becomes

$$
\delta W_{\mathrm{in}}=\delta W_{\mathrm{in}}^{\prime}+\int_{A} \Delta f_{i} \delta u_{i} d A,
$$

where $\delta W_{\mathrm{in}}^{\prime}$ is the work of the remaining incremental forces.

\subsection{Partial pressures in the equilibrium state}

An interesting property of partial pressures $p_{k}$ of the various substances in solution is obtained by considering the variational equilibrium condition (6.12). 
We vary only the masses $\delta m_{k}$ inside $\Omega$. Hence, $\delta W_{e q}^{\prime}=0$. Equation (6.12) becomes

$$
\left.\int_{\Omega}^{k} \sum_{0 k}+\mathscr{G}\left(x_{i}\right)\right] \delta m_{k} d \Omega=0 .
$$

We must satisfy the constraint of mass conservation

$$
\int_{\mathbf{\Omega}} \delta m_{k} d \Omega=0 .
$$

This is satisfied identically by putting $\delta m_{k}=\partial\left(\delta F_{i}^{k}\right) / \partial x_{i}$ where $\delta F_{i}^{k}$ are arbitrary vectors. Substitution of this value of $\delta m_{k}$ in the integral (6.22) and integration by parts yields

$$
\frac{\partial}{\partial x_{i}}\left[\psi_{0 k}+\mathscr{G}\left(x_{i}\right)\right]=0
$$

or

$$
\frac{1}{\rho_{0 k}} \frac{\partial p_{0 k}}{\partial x_{i}}+\frac{\partial \mathscr{G}}{\partial x_{i}}=0
$$

where $p_{0 k}$ is the partial pressure of substance $k$ in the initial state of equilibrium at the temperature $T_{0}$ and $\rho_{0 k}$ is its corresponding density in the pure state. Hence, isobaric surfaces of partial pressures in the equilibrium state coincide with equipotential surfaces $\mathscr{G}=$ const.

\section{Entropy Production and the Princtple of Virtual Disstipation}

The initially-stressed continuum is a thermodynamic system in equilibrium in its initial state. Small perturbations obey the principles of linear thermodynamics (BIOT, 1955). It was shown that the virtual dissipation may be written (with the summation convention)

$$
T_{0} \delta S^{\prime}=\frac{\partial D}{\partial \dot{q}_{i}} \delta q_{i}
$$

where $q_{i}$ is any extensive state variable satisfying the fundamental energy and mass transport constraints as well as the mechanical constraints. The invariant $D$ is a quadratic form in the time-derivatives $\dot{q}_{i}$. This formulation embodies ONSAGER's (1931) principle. The rate of entropy production is

$$
\dot{S}^{\prime}=\frac{2}{T_{0}} D
$$

Following d'Alembert's principle we may include the reversed inertia forces as body forces. The virtual work (6.21) is then

$$
\delta W_{\text {in }}=-I_{i} \delta q_{i}+Q_{i}^{\prime} \delta q_{i},
$$

where $I_{i}$ are generalized inertia forces and

$$
Q_{i}^{\prime} \delta q_{i}=\int_{A} \Delta f_{i} \delta u_{i} d A
$$


is the virtual work of the incremental boundary tractions $\Delta f_{i}$ per unit initial area.

The variational equation (6.19) becomes

$$
\delta \mathscr{P}+\frac{\partial D}{\partial \dot{q}_{i}} \delta q_{i}+I_{i} \delta q_{i}=Q_{i}^{\prime} \delta q_{i}
$$

Equation (7.5) constitutes the basic variational principle of linear thermodynamics (BIOT, 1955). This principle was recently generalized in the same form for non-linear irreversible thermodynamics and referred to as the principle of virtual dissipation (B1OT, 1975, 1976b).

In order to apply this variational principle we must define the variables to be varied. These variables are the material displacement vector $u_{i}$, the mass displacement vectors $M_{i}^{k}$, and an entropy displacement vector $S_{i}^{T}$ satisfying the holonomic constraints

$$
a_{i j}=\partial u_{i} / \partial x_{j}, \quad m_{k}=-\partial M_{i}^{k} / \partial x_{i}, \quad s_{T}=-\partial S_{i}^{T} / \partial x_{i} .
$$

The rate of mass flow per unit area for each substance relative to the solid is $\dot{M}_{i}^{k}$ (BIOT, 1970, p. 162). The entropy displacement (BIOT, 1970, p. 167) is defined by the relation

$$
\dot{S}_{i}^{T}=\dot{H}_{i} / T_{0},
$$

where $\dot{H}_{i}$ is the rate of heat flow due to conduction per unit area. If $\dot{s}^{*}$ denotes the rate of entropy production per unit volume, then the local dissipation function per unit volume is

$$
\mathscr{D}_{\mathrm{d}}=\frac{1}{2} T_{0} \dot{s}^{*}
$$

This is a quadratic form in the local rate variables $\dot{M}_{i}^{k}$ and $\dot{S}_{i}^{T}$, and hence

$$
\mathscr{D}_{\mathrm{d}}=\frac{1}{2} \sum^{k} \mathscr{C}_{i j}^{l k} \dot{M}_{i}^{k} \dot{M}_{j}^{l}+\sum^{k} \mathscr{C}_{i j}^{k} \dot{M}_{i}^{k} S_{j}^{T}+\frac{1}{2} T_{0} \lambda_{i j} \dot{S}_{i}^{T} \dot{S}_{j}^{T}
$$

The subscript $d$ indicates that it represents the dissipation due to thermomolecular diffusion. The symmetry properties of the coefficients are evident from the nature of the quadratic form.

The term $\lambda_{i j} \dot{S}_{i}^{T} \dot{S}_{j}^{T}$ represents the rate of entropy production due to thermal conduction in the absence of mass flow $\left(\dot{M}_{i}^{k}=0\right.$ ), (MEIXNER, 1941). The tensor $\lambda_{i j}$ is the inverse of the local thermal conductivity.

The total dissipation function of the continuum is

$$
D=\int_{\Omega} \mathscr{D}_{\mathrm{d}} d \Omega \text {. }
$$

An important property of linear systems with small perturbations results from the fact that the entropy produced $s^{*}$ is a second-order quantity. Therefore its contribution to the state variables is negligible. Hence, $u_{i}, M_{i}^{k}$ and $S_{i}^{T}$ completely describe the state of the continuum to the first order.

\section{Field Equations Derived from the Variational Principle}

The unknown fields to be determined are $u_{i}, M_{i}^{k}$ and $S_{i}^{T}$. The dynamic differential field equations which govern these unknown vectors are obtained directly from the 
variational principle (7.5) by varying $u_{i}, M_{i}^{k}$ and $S_{i}^{T}$ arbitrarily inside the domain $\Omega$.

The variation of $(6.20)$ is

$$
\delta \mathscr{P}=\int_{\Omega}\left(\delta v+S_{i j} \delta \eta_{i j}+\Delta K_{i} \delta u_{i}+\sum^{k} \mathscr{G}_{i} u_{i} \delta m_{k}\right) d \Omega
$$

where

$$
\Delta K_{i}=\rho \mathscr{G}_{i j} u_{j}+\sum^{k} \mathscr{G}_{i} m_{k} .
$$

From (5.6), noting that $2 \delta e_{i j}=\delta\left(a_{i j}+a_{j i}\right)$, we may write

$$
\delta v=t_{i j} \delta a_{i j}+\sum^{k} \Delta \psi_{k} \delta m_{k}+\theta \delta s_{T} .
$$

Using the holonomic constraints (7.6) and integrating by parts, we obtain

$$
\delta \mathscr{P}=\int_{\mathbf{\Omega}}\left[\left(-Y_{i}+\Delta K_{i}\right) \delta u_{i}+\sum^{k} \frac{\partial}{\partial x_{i}}\left(\Delta \phi_{k}\right) \delta M_{i}^{k}+\frac{\partial \theta}{\partial x_{i}} \delta S_{i}^{T}\right] d \Omega
$$

with

$$
Y_{i}=\frac{\partial}{\partial x_{j}}\left(t_{i j}+S_{\mu v} \frac{\partial \eta_{\mu v}}{\partial a_{i j}}\right), \quad \Delta \phi_{k}=\Delta \psi_{k}+\mathscr{G}_{i} u_{i}
$$

The virtual dissipation is

$$
\frac{\partial D}{\partial \dot{q}_{i}} \delta q_{i}=\int_{\Omega}\left(\sum^{k} \frac{\partial \mathscr{D}_{\mathrm{d}}}{\partial \dot{M}_{i}^{k}} \delta M_{i}^{k}+\frac{\partial \mathscr{D}_{\mathrm{d}}}{\partial \dot{S}_{i}^{T}} \delta S_{i}^{T}\right) d \Omega
$$

The virtual work of the inertia forces is

$$
I_{i} \delta q_{i}=\int_{\Omega}^{ \pm}\left[\left(\rho \dot{u}_{i}{ }^{k} \delta u_{i}+\sum^{k} m_{0 k}\left(\ddot{u}_{i}+a_{i}^{k}\right) \delta u_{i}^{k}\right] d \Omega .\right.
$$

The initial mass of substance $k$ per unit initial volume is $m_{0 k}, \delta u_{i}^{k}$ is the virtual displacement of the substance due to $\delta M_{i}^{k}$, and $a_{i}^{k}$ is the acceleration of the substance relative to the solid in which it is dissolved. We may write

$$
\delta u_{i}^{k}=\delta M_{i}^{k} / m_{0 k}, \quad a_{i}^{k}=\ddot{M}_{i}^{k} / m_{0 k} .
$$

By introducing the kinetic energy per unit volume,

$$
\mathscr{E}=\frac{1}{2} \rho \dot{u}_{i}^{2}+\sum^{k} \dot{u}_{i} \dot{M}_{i}^{k}+\frac{1}{2} \sum^{k} \dot{M}_{i}^{k} \dot{M}_{i}^{k} / m_{0 k}
$$

the virtual work (8.6) of the inertia forces becomes

$$
I_{i} \delta q_{i}=\int_{\Omega}\left[\frac{d}{d t}\left(\frac{\partial \mathscr{E}}{\partial \dot{u}_{i}}\right) \delta u_{i}+\sum \frac{d}{d t}\left(\frac{\partial \mathscr{E}}{\partial \dot{M}_{i}^{k}}\right) \delta \mathscr{H}_{i}^{k}\right] d \Omega .
$$

We substitute the values (8.3), (8.5) and (8.9) of $\delta \mathscr{P},\left(\partial D / \partial \dot{q}_{i}\right) \delta q_{i}$ and $I_{i} \delta q_{i}$ in the variational principle (7.5), and vary arbitrarily $\delta u_{i}, \delta M_{i}^{k}$ and $\delta S_{i}^{T}$ inside $\Omega$. Hence the term $Q_{i}^{\prime} \delta q_{i}$ at the boundary vanishes. By equating to zero the coefficients of 
$\delta u_{i}, \delta M_{i}^{k}$ and $\delta S_{i}^{T}$ we obtain the dynamical field equations

$$
\left.\begin{array}{r}
\frac{d}{d t}\left(\frac{\partial \mathscr{E}}{\partial \dot{u}_{i}}\right)-\frac{\partial}{\partial x_{j}}\left(t_{i j}+S_{\mu v} \frac{\partial \eta_{\mu v}}{\partial a_{i j}}\right)+\Delta K_{i}=0 \\
\frac{d}{d t}\left(\frac{\partial \mathscr{E}}{\partial \dot{M}_{i}^{k}}\right)+\frac{\partial}{\partial x_{i}}\left(\Delta \phi_{k}\right)+\frac{\partial \mathscr{D}_{\mathrm{d}}}{\partial \dot{M}_{i}^{k}}=0 \\
\frac{\partial \theta}{\partial x_{i}}+\frac{\partial \mathscr{D}_{\mathrm{d}}}{\partial \dot{S}_{i}^{T}}=0
\end{array}\right\}
$$

These equations govern acoustic propagation with initial stress and thermomolecular diffusion. They are linear in the unknown field components $u_{i}, M_{i}^{k}$ and $S_{i}^{T}$. They are valid for a nonhomogeneous continuum, non-uniform initial stress and body force field. In this case, the coefficients are functions of the initial coordinates.

The last two groups of equations containing $\mathscr{D}_{\mathrm{d}}$ govern the thermomolecular diffusion for which the driving forces are the inertia force $(d / d t)\left(\partial \mathscr{E} / \partial \dot{M}_{i}^{k}\right)$, the temperature gradient $\partial \theta / \partial x_{i}$, and the gradient $\left(\partial / \partial x_{i}\right) \Delta \phi_{k}$ of $\Delta \phi_{k}$. According to (8.4),

$$
\frac{\partial}{\partial x_{i}}\left(\Delta \phi_{k}\right)=\Delta \frac{\partial}{\partial x_{i}}\left(\psi_{k}+\mathscr{G}\right)
$$

where $\Delta \mathscr{G}=\mathscr{G}_{i} u_{i}$ is the increase of body force potential $\mathscr{G}$ when we follow the material displacement $u_{i}$ in the potential field $\mathscr{G}$. Hence, the driving force (8.11) is the increase of gradient of the mixed potential $\psi_{k}+\mathscr{G}$. According to (6.24), we verify that this driving force vanishes in the initial state of equilibrium.

\section{Lagrangiań Equations Derived from the Variational Principle}

We note that the differential field equations (8.10) are in a Lagrangian form. In fact, the field variables may be considered as a particular case of generalized coordinates. Completely general Lagrangian equations may be derived directly from the variational principle (7.5). We write the unknown fields in the form

$$
\left.\begin{array}{rl}
u_{i} & =u_{i}\left(q_{1}, q_{2}, \ldots, q_{n}, x_{i}\right), \\
M_{i}^{k} & =M_{i}^{k}\left(q_{1}, q_{2}, \ldots, q_{n}, x_{l}\right), \\
S_{i}^{T} & =S_{i}^{T}\left(q_{1}, q_{2}, \ldots, q_{n}, x_{l}\right),
\end{array}\right\}
$$

where $q_{i}$ are generalized coordinates. In particular they may be of the 'penetration depth' type (Biot, 1970). If need be, expressions (9.1) may also include the time explicitly; however, for simplicity, we shall assume that this is not the case. The collective potential $\mathscr{P}$ is now a function of $q_{i}$ :

$$
\mathscr{P}=\mathscr{P}\left(q_{i}\right) \text {. }
$$

The total dissipation function (7.10) is

$$
D=\int_{\Omega} \mathscr{D}_{\mathrm{d}} d \Omega=\frac{1}{2} B_{i j} \dot{q}_{i} \dot{q}_{j}
$$

and the total kinetic energy is

$$
\mathscr{T}=\int_{\Omega} \mathscr{E} d \Omega=\frac{1}{2} T_{i j} \dot{q}_{i} \dot{q}_{j} .
$$

The coefficients $B_{i j}$ and $T_{i j}$ are functions of $q_{i}$. 
An important particular case is obtained by choosing for the representation (9.1) linear functions of $q_{i}$ with coefficients which are functions of the coordinates $x_{i}$. In this case, $D$ and $\mathscr{T}$ become quadratic forms in $\dot{q}_{i}$ with constant coefficients $B_{i j}$ and $T_{i j}$. The collective potential also becomes a quadratic form in $q_{i}$

$$
\mathscr{P}=\frac{1}{2} A_{i j} q_{i} q_{j}
$$

with constant coefficients $A_{i j}$.

The variational principle (7.5) assumes that the fields $M_{i}^{k}$ and $S_{i}^{T}$ do not vary at the boundary of $\Omega$. In order to avoid this restriction, following a procedure already used many times earlier (Віот, 1955, 1973, 1975), we adjoin to the system driving cells at the boundary and we consider them to be part of the domain $\Omega$. These driving cells provide a contribution $\mathscr{P}^{\prime}$ to the collective potential such that

$$
\delta \mathscr{P}^{\prime}=\int_{\boldsymbol{A}}\left(\sum^{k} \Delta \phi_{k} \frac{\partial M_{j}^{k}}{\partial q_{i}}+\theta \frac{\partial S_{j}^{T}}{\partial q_{i}}\right) n_{j} \delta q_{i} d A,
$$

where the surface integral is over the boundary of the continuum. In the variational principle (7.5) we may then replace $\mathscr{P}$ by $\mathscr{P}+\mathscr{P}$ '. By a classical procedure we also obtain the virtual work of the inertia forces in the form

$$
I_{i} \delta q_{i}=\left[\frac{d}{d t}\left(\frac{\partial \mathscr{T}}{\partial \dot{q}_{i}}\right)-\frac{\partial \mathscr{T}}{\partial q_{i}}\right] \delta q_{i}
$$

Hence, the variational principle (7.5) leads to the Lagrangian equations

where

$$
\frac{d}{d t}\left(\frac{\partial \mathscr{T}}{\partial \dot{q}_{i}}\right)-\frac{\partial \mathscr{T}}{\partial q_{i}}+\frac{\partial D}{\partial \dot{q}_{i}}+\frac{\partial \mathscr{P}}{\partial q_{i}}=Q_{i}
$$

$$
Q_{i}=-\frac{\partial \mathscr{P}^{\prime}}{\partial q_{l}}+Q_{i}^{\prime}=\int_{A}\left(\Delta f_{j} \frac{\partial u_{j}}{\partial q_{i}}-\sum \Delta \phi_{k} \frac{\partial M_{j}^{k}}{\partial q_{i}} n_{j}-\theta \frac{\partial S_{j}^{T}}{\partial q_{i}} n_{j}\right) d A
$$

represents a generalized boundary driving force of mixed mechanical and thermodynamic nature. It generalizes similar expressions obtained earlier (BIoT, 1973, 1975). Numerous techniques have been developed for the solution of complex problems by these Lagrangian methods (BIOT, 1970, LARDNER, 1967, CHUNG and Yre, 1975, Prasad and Agrawal, 1972, 1974, and Yeh, 1976). When the fields (9.1) are chosen as linear functions of the generalized coordinates $q_{i}$, the Lagrangian equations become linear:

$$
\frac{d}{d t}\left(\frac{\partial \mathscr{T}}{\partial \dot{q}_{i}}\right)+\frac{\partial D}{\partial \dot{q}_{i}}+\frac{\partial \mathscr{P}}{\partial q_{i}}=Q_{i}
$$

with the same constant coefficients as in the quadratic forms (9.3), (9.4) and (9.5).

We may of course express these results in Hamiltonian form by writing the variational principle as

$$
\int_{o}^{t}\left[\delta(\mathscr{T}+\mathscr{P})+\frac{\partial D}{\partial \dot{q}_{i}} \delta q_{i}-Q_{i} \delta q_{i}\right] d t=0,
$$

where the integration is with respect to the time $t$. 


\subsection{Reciprocity relations}

In the linear equations (9.10), the coefficient matrices are symmetric. As a consequence, the response $q_{j}$ to a force $Q_{i}$ is equal to the response $q_{i}$ due to a force $Q_{j}$ equal to $Q_{i}$. This basic reciprocity property is valid for all linear thermodynamic systems and does not require a new proof for each particular problem and for each set of boundary conditions. Note that $q_{i}$ and $Q_{i}$ are quite general and include local fields and forces.

\section{Extension to a Solid with Chemical Reactions}

Consider a cell where several chemical reactions may occur. Masses of the various substances produced per unit cell volume are expressed by

$$
d m_{k}=\sum^{\rho} v_{k \rho} d \xi_{\rho}
$$

where $\xi_{\rho}$ are the coordinates of the various reactions. Mass conservation implies

$$
\sum v_{k \rho}=0 .
$$

For a forward reaction $\left(d \xi_{\rho}>0\right)$, some masses 'produced' are positive, and some are negative. The pure substances in the reaction may be less or larger in number than the substances exchanged by the diffusion.

Following an earlier derivation (BroT, 1976a,1977), we consider reactions taking place in a rigid adiabatic closed cell. Since the energy of the cell does not vary ( $d U$ $=0$ ), it follows from (2.3) that the change in cell potential is

$$
d \mathscr{V}_{\text {ch }}=-T_{0} d \mathscr{S}_{\text {ch, }}
$$

where $d \mathscr{S}_{\text {ch }}$ is the entropy produced by the reactions. We put

$$
d \mathscr{S}_{\mathrm{ch}}=\sum^{\rho} \frac{A_{\rho}}{T_{0}} d \xi_{\rho}
$$

where $A_{\rho}$ are the affinities as defined by De Donder (1936). At chemical equilibrium, $A_{\rho}=0$ and we choose $\xi_{\rho}=0$. Hence, $A_{\rho}$ and $\xi_{\rho}$ are first-order quantities. Equation (10.2) becomes

$$
d \mathscr{V}_{\mathrm{ch}}=-\sum^{\rho} A_{\rho} d \xi_{\rho}
$$

For a cell which is open and neither rigid nor adiabatic while at the same time undergoing chemical reactions, the change of incremental cell potential is the sum of expressions (10.4) and (5.6); and hence

$$
d v=-\sum^{\rho} A_{p} d \xi_{p}+t_{i j} d e_{i j}+\sum^{k} \Delta \psi_{k} d M^{k}+\theta d s_{T} .
$$

We have replaced the $m_{k}$ by $M^{k}$ to indicate that they represent the masses acquired by diffusion as distinct from the $v_{k \rho} \xi_{\rho}$ which are produced by the chemical reactions. The state variables of the cell are now $\xi_{\rho}, e_{i j}, M^{k}$ and $s_{T}$. The incremental potential $v$ is a quadratic form in these variables. From (10.5) we derive

$$
\partial v / \partial \xi_{\rho}=-A_{\rho}, \quad \partial v / \partial e_{i j}=t_{i j}, \quad \partial v / \partial M^{k}=\Delta \psi_{k}, \quad \partial v / \partial s_{T}=\theta
$$


By choosing $\xi_{\rho}, e_{i j}, M^{k}$ and $\theta$ as state variables we have the linear relation

$$
T_{0} s_{T}=\gamma_{i j} e_{i j}+\sum^{k} h^{k} M^{k}+\sum^{\rho} h_{\rho} \xi_{\rho}+c \theta
$$

which generalizes (5.11) and where $h_{\rho}$ is now the heat of each reaction for $e_{i j}=M_{k}=\theta=0$.

A simple way to evaluate $v$ and the affinity is obtained by considering the system composed of a primary cell $C_{\mathrm{p}}$ with the potential $v$ and a reference chemical cell $C_{\mathrm{ch}}$ with a potential $v^{\prime}$. The latter is assumed to be in equilibrium so that $v^{\prime}=0$. Masses $v_{k \rho} \xi_{\rho}$ are transferred from $C_{\mathrm{ch}}$ to $C_{\mathrm{p}}$ by thermobaric transfer while the state of $C_{\mathrm{ch}}$ remains unchanged by allowing the chemical reaction to supply the masses extracted. Thus, $v^{\prime}$ remains equal to zero and the total potential change is $v+v^{\prime}=v$. In this process, we assume that we maintain constant the temperature $(\theta=0)$ in both cells. The state of the system obviously depends only on the masses $m_{k}$ contained in $C_{\mathrm{p}}$ whether they are the result of chemical reactions or thermobaric transfer. Hence, the value (5.8) of $\bar{v}$ is valid with chemical reactions provided we replace $m_{k}$ by

$$
m_{k}=\sum^{\rho} v_{k \rho} \xi_{\rho}+M^{k}
$$

When the temperature is also varied, the potential is equal to (5.10):

$$
v=\bar{v}+\frac{1 c \theta^{2}}{2}
$$

According to equations (10.6), the affinities are given by

$$
-A_{\rho}=\frac{\partial v}{\partial \xi_{\rho}}=\sum \frac{\partial \bar{v}}{\partial m_{k}} \frac{\partial m_{k}}{\partial \xi_{\rho}}+\frac{c \theta}{T_{0}} \frac{\partial \theta}{\partial \xi_{\rho}}
$$

The partial derivatives $\partial m_{k} / \partial \xi_{\rho}$ and $\partial \theta / \partial \xi_{\rho}$ are obtained from (10.7) and (10.8). Hence,

$$
-A_{\rho}=\sum v_{k \rho}^{k} \frac{\partial \vec{v}}{\partial m_{k}}-\frac{\theta h_{\rho}}{T_{0}}
$$

The derivative $\partial \bar{v} / \partial m_{k}$ is given by expression (5.13) whose physical significance has already been discussed. We thus obtain for $A_{p}$ the linear expression (with the value (10.8) for $m_{l}$ )

$$
A_{\rho}=-\sum^{k} v_{k \rho} C_{i j}^{k} e_{i j}-\sum^{k l} C^{k l} v_{k \rho} m_{l}+\frac{\theta h_{\rho}}{T_{0}} .
$$

It remains to relate $A_{\rho}$ to the reaction rates $\dot{\xi}_{\rho}$. The Onsager principle leads to the dissipation function

$$
\mathscr{D}_{\mathrm{ch}}=T_{0} \dot{s}_{\mathrm{ch}}^{*}=\frac{1}{2} \sum^{\rho \sigma} \mathscr{B}_{\rho \sigma} \dot{\xi}_{\rho} \dot{\xi}_{\sigma},
$$

where $\dot{s}_{\mathrm{ch}}^{*}$ is the rate of entropy production by the chemical reactions and $\mathscr{B}_{\rho \sigma}$ are constant, symmetric, rate coefficients. We have the property

$$
A_{\rho}=\frac{\partial \mathscr{D}_{\mathrm{ch}}}{\partial \dot{\xi}_{\rho}}
$$


The dissipation function for simultaneous thermomolecular diffusion and chemical reactions is

$$
\mathscr{D}=\mathscr{D}_{\mathrm{d}}+\mathscr{D}_{\text {ch }}
$$

Note that the dissipation functions $\mathscr{D}_{\mathrm{d}}$ and $\mathscr{D}_{\mathrm{ch}}$ are uncoupled because the former involves vectors whereas the latter involves scalars. This is in accordance with Curie's symmetry principle.

Using (10.9) and (10.7), the value of $v$ is derived as a quadratic form in $\xi_{\rho}, e_{i j}$, $M^{k}$ and $s_{T}$. Application of the variational principle, as in Section 7, leads to the field equations

$$
\left.\begin{array}{r}
\frac{d}{d t}\left(\frac{\partial \mathscr{E}}{\partial \dot{u}_{i}}\right)-\frac{\partial}{\partial x_{j}}\left(t_{i j}+S_{\mu v} \frac{\partial \eta_{\mu D}}{\partial a_{i j}}\right)+\Delta K_{i}=0 \\
\frac{d}{d t}\left(\frac{\partial \mathscr{E}}{\partial \dot{M}_{i}^{k}}\right)+\frac{\partial}{\partial x_{i}} \Delta \phi_{k}+\frac{\partial \mathscr{D}}{\partial \dot{M}_{i}^{k}}=0 \\
\frac{\partial \theta}{\partial x_{i}}+\frac{\partial \mathscr{D}}{\partial \dot{S}_{i}^{T}}=0 \\
-A_{\rho}+\frac{\partial \mathscr{D}}{\partial \dot{\xi}_{p}}=0 .
\end{array}\right\}
$$

Lagrangian equations (9.8) are also obtained by adding to (9.1) a representation of $\xi_{\rho}$ by means of the generalized coordinates

$$
\xi_{\rho}=\xi_{\rho}\left(q_{1}, q_{2}, \ldots, q_{n}, x_{l}\right)
$$

and introducing a dissipation function

$$
D=\int_{\mathbf{\Omega}} \mathscr{D} d \mathbf{\Omega}
$$

where $\mathscr{D}$ is given by $(10.15)$ and includes the chemical dissipation.

\section{Acoustic Propagation with Chemical and Other Relaxation Effects}

According to (10.6) and (10.14) we may write

$$
\left.\begin{array}{ccc}
\frac{\partial v}{\partial e_{i j}}=t_{i j}, & \frac{\partial v}{\partial M^{k}}=\Delta \psi_{k}, & \frac{\partial v}{\partial s_{T}}=\theta \\
\frac{\partial v}{\partial \xi_{\rho}}+\frac{\partial \mathscr{D}_{\mathrm{ch}}}{\partial \dot{\xi}_{\rho}}=0 . &
\end{array}\right\}
$$

These equations have the form

$$
\frac{\partial v}{\partial q_{i}}=Q_{i}, \quad \frac{\partial v}{\partial \xi_{\rho}}+\frac{\partial \mathscr{D}_{\mathrm{ch}}}{\partial \dot{\xi}_{\rho}}=0
$$

where $Q_{i}$ represents the driving forces $t_{i j}, \Delta \psi_{k}$ and $\theta$, and $q_{i}$ represents the conjugate variables $e_{i j}, M^{k}$ and $s_{T}$. The potential $v$ is a quadratic form in $q_{i}$ and $\xi_{\rho}$ while $\mathscr{D}_{\text {ch }}$ is a quadratic form in $\xi_{\rho}$. Equations (11.2) are Lagrangian equations derived and discussed in the general context of linear thermodynamics and represent a system 
with internal coordinates $\xi_{\rho}$. It was shown (BIOT, 1954, 1970) that the other coordinates $q_{i}$ are related to the driving forces $Q_{i}$ by the operational relations

$$
Q_{i}=\left(\sum^{s} D_{i j}^{s} \frac{p}{p+r_{s}}+D_{i j}\right) q_{j}
$$

where $p=d / d t$ is the time-derivative operator. The coefficients $D_{i j}^{s}$ and $D_{i j}$ are symmetric and positive definite. The relaxation constants $r_{s}$ are positive. For harmonic time-dependence proportional to $\exp (i \omega t)$, the value of $p$ is $i \omega$. The operational equations (11.3) may also be interpreted as Laplace transforms. For the general case, the operational expression represents an integral operator

$$
\frac{p}{p+r_{s}} f(t)=\int_{0}^{t} \exp \left[r_{s}\left(t^{\prime}-t\right)\right] d f\left(t^{\prime}\right)
$$

Equations (11.3) are integro-differential relations between the driving forces $t_{i j}$, $\Delta \psi_{k}, \theta$ on the one hand and the response variables $e_{i j}, M^{k}, s_{T}$ on the other hand. We may write them, symbolically, as

$$
\left[\begin{array}{c}
t_{i j} \\
\Delta \psi_{k} \\
\theta
\end{array}\right]=[Z]\left[\begin{array}{c}
e_{i j} \\
M^{k} \\
s_{T}
\end{array}\right]
$$

where $[Z]$ is the operational matrix of (11.3), and

$$
e_{i j}=\frac{1}{2}\left(\frac{\partial u_{i}}{\partial x_{j}}+\frac{\partial u_{j}}{\partial x_{i}}\right), \quad M^{k}=-\frac{\partial M_{i}^{k}}{\partial x_{i}}, \quad s_{T}=-\frac{\partial S_{i}^{T}}{\partial x_{i}}
$$

These relations represent a generalized form of viscoelasticity which are essentially the same as those introduced more specifically in the mechanics of viscoelastic solids (BIot, 1954, 1965). The viscoelasticity in the present case is due to chemical relaxation.

Substitution of the values (11.5) in (8.10) yield the acoustic propagation equations under initial stress with thermomolecular diffusion and explicit chemical relaxation effects.

Viscoelastic correspondence introduced as a consequence of irreversible thermodynamics (Bıот, 1954, 1955, 1965) is confirmed here in the same general context; namely, solutions of (8.10) without chemical relaxations are immediately extended to include these simply by replacing the matrix $[Z]$ of constant coefficients by the operators. It is important to point out the extreme generality of this procedure. It may be applied, for example, to the Lagrangian equations (9.10) including explicitly the constant coefficients representing the matrix [Z] without chemical relaxation and then replacing the coefficients by the corresponding operators. This should be extremely useful in the solution of seismic problems.

\subsection{Other relaxation effects}

A large variety of relaxation effects other than chemical ones, such as microscopic phase changes, intergranular viscosities, etc., lead to the same type of equations with suitable internal coordinates. 


\section{REFERENCES}

Biot, M. A.

Chung, B. T. F. and YEH, L. T.

De DONDER, Th.

LARDNER, T. J.

MEIXNER, J.

Mindin, R. D.

NunZiato, J. W.

ONSAGER, L.

Prasad, A. and

Agrawal, H. C.

YEH, L. T.
$1954 \quad J$. appl. Phys. 25, 1385.

1955 Phys. Rev. 97, 1463.

1965 Mechanics of Incremental Deformations. Wiley, New York.

1970 Variational Principles in Heat Transfer. Oxford University Press.

1973 Indiana Univ. math. J. 4, 309.

$1974 \quad$ Int. J. Solids Struct. 10, 419.

1975 Bull. Acad. roy. Belg. Cl. Sci. 61, 6.

1976a Ibid. 62, 239.

1976b Q. appl. Math. 34, 213.

1977 Chem. Phys. 22, 183.

1975 J. Spacecraft Rockets 12, 329.

1934 L'Affinité. Gauthiers-Villars, Paris.

1967 ALAA J. 5, 2079.

1941 Annln Phys. 39, 333.

1961 Problems of Continuum Mechanics (Contributions in Honor of the Seventieth Birthday of Academician N. I. Muskhelishvili, 16th February 1961; edited by Lavrent'ev, M. A., et $a l$.), (English edition edited by Radok, J. R. M.), p. 282. Society for Industrial and Applied Mathematics, Philadelphia.

1974 Int. J. Solids Struct. 10, 625.

1973 J. Chem. Phys. 58, 961.

1931 Phys. Rev. 37, 405; 38, 2265.

$1972 \quad$ AIAA J. 10, 325.

1974 Ibid. 12, 250.

1975 On Biot's variational method for some nonlinear transport processes involving thermal radiation. Ph.D. Dissertation, University of Akron. 